Portland State University

PDXScholar

9-15-2014

\title{
Positional Control of Plasmonic Fields and Electron Emission
}

\author{
Robert Campbell Word \\ Portland State University, wordr@pdx.edu \\ Joseph P. Fitzgerald \\ Portland State University, fit@pdx.edu \\ Rolf Könenkamp \\ Portland State University, rkoe@pdx.edu
}

Follow this and additional works at: https://pdxscholar.library.pdx.edu/phy_fac

Part of the Plasma and Beam Physics Commons

Let us know how access to this document benefits you.

\section{Citation Details}

Word, R. C., Fitzgerald, J. P. S., \& Könenkamp, R. (2014). Positional control of plasmonic fields and electron emission. Applied Physics Letters, 105(11), 111114.

This Article is brought to you for free and open access. It has been accepted for inclusion in Physics Faculty Publications and Presentations by an authorized administrator of PDXScholar. Please contact us if we can make this document more accessible: pdxscholar@pdx.edu. 


\section{AIP Applied Physics \\ Letters}

\section{Positional control of plasmonic fields and electron emission}

R. C. Word, J. P. S. Fitzgerald, and R. Könenkamp

Citation: Applied Physics Letters 105, 111114 (2014); doi: 10.1063/1.4896111

View online: http://dx.doi.org/10.1063/1.4896111

View Table of Contents: http://scitation.aip.org/content/aip/journal/apl/105/11?ver=pdfcov

Published by the AIP Publishing

\section{Articles you may be interested in}

Optical spin-to-orbital angular momentum conversion in ultra-thin metasurfaces with arbitrary topological charges Appl. Phys. Lett. 105, 101905 (2014); 10.1063/1.4895620

Bidirectional waveguide coupling with plasmonic Fano nanoantennas

Appl. Phys. Lett. 105, 053114 (2014); 10.1063/1.4892651

Mapping near-field localization in plasmonic optical nanoantennas with $10 \mathrm{~nm}$ spatial resolution Appl. Phys. Lett. 105, 053105 (2014); 10.1063/1.4892577

Ultra-sharp plasmonic resonances from monopole optical nanoantenna phased arrays Appl. Phys. Lett. 104, 231101 (2014); 10.1063/1.4881323

Photoelectron emission control with polarized light in plasmonic metal random structures Appl. Phys. Lett. 99, 041106 (2011); 10.1063/1.3615783

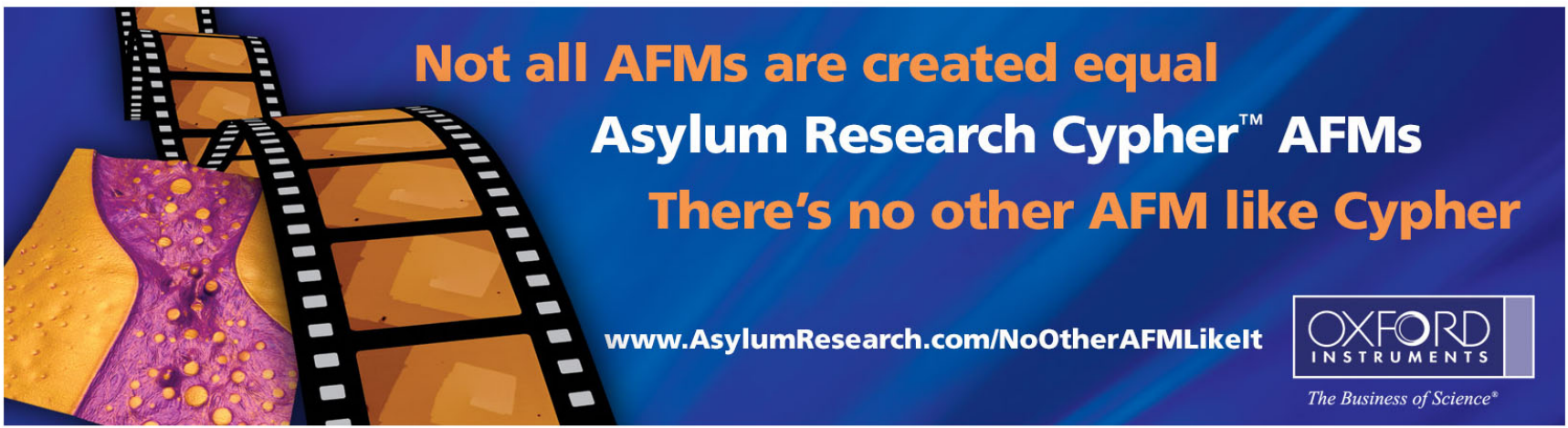




\title{
Positional control of plasmonic fields and electron emission
}

\author{
R. C. Word, J. P. S. Fitzgerald, and R. Könenkamp ${ }^{\text {a) }}$ \\ Department of Physics, Portland State University, 1719 SW 10th Avenue, Portland, Oregon 97201, USA
}

(Received 12 August 2014; accepted 6 September 2014; published online 19 September 2014)

\begin{abstract}
We report the positional control of plasmonic fields and electron emission in a continuous gap antenna structure of sub-micron size. We show experimentally that a nanoscale area of plasmonenhanced electron emission can be motioned by changing the polarization of an exciting optical beam of $800 \mathrm{~nm}$ wavelength. Finite-difference calculations are presented to support the experiments and to show that the plasmon-enhanced electric field distribution of the antenna can be motioned precisely and predictively. (C) 2014 AIP Publishing LLC.

[http://dx.doi.org/10.1063/1.4896111]
\end{abstract}

Plasmonic enhancement of optical fields is currently widely explored for applications in sensing, energy conversion, information transfer, and in microscopy. ${ }^{1-6}$ Localized plasmon resonances have been realized in planar gap-type antenna structures, in nanometer-sized tip configurations and in tip-substrate cavities. All of these configurations are capable of confining large electric field strengths in very small regions. In a recent breakthrough, Zhang et al. ${ }^{7}$ showed that a plasmonically coupled STM tip is capable of resolving intramolecular features of a mid-sized organic molecule. This extraordinary possibility of confining optical fields to nanometer spaces is important for sensing and for many microscopic and spectroscopic applications. As a next step in this development, it is desirable to motion the confined field region either to fine-tune the spatial overlap between probing field and sample, to carry out spatial scanning procedures or to selectively address separate receivers in routing applications. ${ }^{8}$

In this paper, we present a sub-micron device that allows us to precisely position localized plasmon resonances using polarization control in the optical excitation beam. Essentially, by polarization control a specific orientation among the antenna modes in a ring-shaped antenna is selected and by changing the polarization vector the nodes are motioned towards a specific region of the antenna gap. Both the optical field strength distribution and the accompanying non-linear photoelectron emission are monitored with an aberration-corrected photoemission electron microscope using a femtosecond laser pulses as an excitation source. We show experimental results for the spatial control of the localized surface plasmon resonances and the electron emission. The experiments are supported by detailed finite-element calculations.

We use a ring-shaped continuous gap structure with a circular groove of $300 \mathrm{~nm}$ width milled into a copper/gold bi-layer to generate electron emission from an underlying indium-tin-oxide (ITO) layer that is exposed by focused-ionbeam milling. Fig. 1 shows a schematic of the used structure. A $310 \mathrm{~nm}$ thick ITO film on glass substrate is covered with an evaporated $50 \mathrm{~nm}$ thick $\mathrm{Cu}$ layer and a $50 \mathrm{~nm}$ thick $\mathrm{Au}$ layer. The $\mathrm{Cu} / \mathrm{Au}$ bi-layer arrangement is used here, because it can be structured with better precision than a single gold layer in our 235 FEI dual-beam Focused Ion Beam machine.

a)E-mail: rkoe@pdx.edu
A circular groove of $300 \mathrm{~nm}$ width and $350 \mathrm{~nm}$ inner diameter is milled through the two metal layers, such that the grove bottom is ITO. A layer of $\sim 160 \mathrm{~nm}$ thickness is milled off the ITO in the exposed area. Illumination is obtained from a Ti-sapphire laser providing $100 \mathrm{fs}$ light pulses at a frequency of $100 \mathrm{MHz}$ and a wavelength of $800 \mathrm{~nm}$. The excitation light falls on the sample at an angle of $60^{\circ}$ from the sample surface normal. The polarization of the laser light can be controlled with a tunable wave-plate. An aberrationcorrected photoemission microscope ${ }^{9}$ is used to image the photoelectron emission from the device. From the photoemission image, the plasmonic field distribution and the electron emission distribution can be determined. For the current work, the spatial resolution is $\sim 45 \mathrm{~nm}$. The calculation of the electric field distribution is carried out in a finite-element approach utilizing the full dielectric functions of the materials and the true spatial dimensions of the device. The calculation was carried out in the RF-Module of COMSOL 4.3, which solves Maxwell's equations for a triangular mesh to obtain the electric field distribution. The mesh size was smaller than $\lambda / 10$, as recommended for this type of model. The calculations were carried out on a dual CPU $2.5 \mathrm{GHz}$ workstation with 180 GB RAM.

The photoelectron emission in this structure is predominantly from the ITO gap region, because of its lower work function: The incident light has a photon energy of $1.5 \mathrm{eV}$. The workfunction of ITO is $4.3 \mathrm{eV}$ and photoemission can occur in a 3-photon process. The efficiency, threshold energy, and non-linear intensity dependence of the electron emission from ITO in this type of experiment have been reported in an earlier paper. ${ }^{10}$ The gold surfaces in the device have a workfunction of $\sim 5 \mathrm{eV}$ and photoemission would require a 4-photon process, which has lower probability. As a consequence, photoemission from areas outside the circular ITO groove is very small. At the wavelength of $800 \mathrm{~nm}$, the ITO/glass substrate is transparent and at the corresponding optical frequency, it essentially acts as a dielectric. The metal-ITO ring structure can therefore be considered as a planar optical antenna. In gold nanostructures, the localized surface plasmon resonance typically lies between 500 and $800 \mathrm{~nm}$ depending on geometry. As the resonance is not very sharp, it can well be excited by the $800 \mathrm{~nm}$ light. The antenna fields are then plasmonically enhanced and acquire very high field strengths in the gap region, i.e., in the ITO groove. ${ }^{11,12}$ 


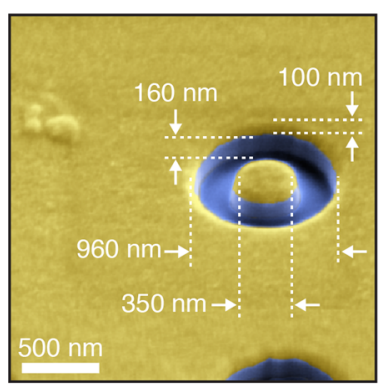

(a)

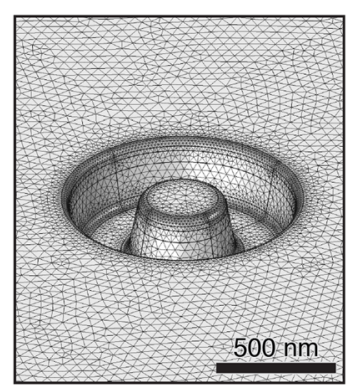

(b)

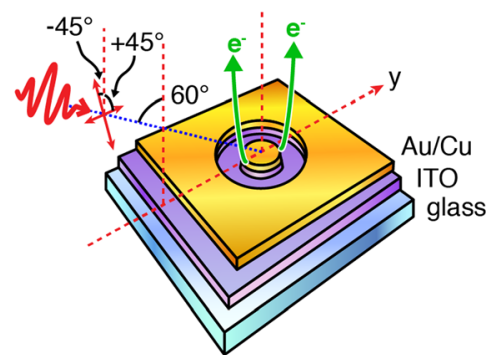

(c)
FIG. 1. Circular-gap antenna structure. (a) Scanning electron micrograph of circular groove milled through a $\mathrm{Au} /$ $\mathrm{Cu}$ bi-layer on ITO-glass; (b) Model structure used in the calculations; (c) Experimental arrangement with light incident at an angle of $60^{\circ}$ from the sample surface normal.
The plasmonic enhancement and the lower work-function of the ITO generate a significant photoelectron emission from the ITO.

The experimental results shown in Fig. 2(a) are obtained with TM polarized light. To compare the image with calculated optical results, we plot the third root of the photoemission yield, $\mathrm{Y}^{1 / 3}$. The image shows dipole and quadrupole-like field distributions of the circular antenna structure in the antenna plane. The predominant contribution of the electron emission originates from the groove area, there is no significant emission from the gold surfaces, as expected and in agreement with earlier findings. ${ }^{10}$ In the azimuthal direction, four peaked emission regions are apparent. The plasmonic response is largest at the far end of the ring structure, where the electric field vector is perpendicular to the center disc edge and the surface curvature is smallest. For these conditions, the in-coupling of optical power and the excitation of surface plasmon resonances are most efficient. For the linear TM polarization used here and the inclined incidence of the optical beam, one expects the plasmonic response to be symmetric to the plane of incidence. The observed quadrupole-like mode distribution and its orientation are in agreement with these expectations. Additional experiments show that the number of emission nodes depends on the relative size of the ring perimeter and wavelength. When the ring perimeter length is increased, more nodes emerge indicating that the structure in the emission pattern is governed by surface plasmon edge modes, as discussed in more detail in Ref. 13. The simulations show that at vertical incidence of the laser excitation the plasmonic mode distribution becomes dipolar, indicating that the broken symmetry for inclined incidence is responsible for the more complex mode distribution.

Fig. 2(b) shows the calculated data for the optical intensity distribution from the finite-element method. The optical intensity, $|\mathrm{E}|^{2}$, is shown, as determined from the electric field strength E. A direct comparison between the photoemission results and the field strength calculation is not entirely straightforward, as workfunction, electron surface density, electric field direction, and tunneling probability are factors contributing to the photoemission rate. In addition, the ITO surface is curved at the edges of the milled area. A good interpretation of the observed photoelectron image can nonetheless be given: In a 3-photon process, the photoemission yield is approximately proportional to the 3rd power of the optical intensity, ${ }^{14}$ which itself is proportional to the square of the field strength as calculated in COMSOL. Therefore, the third root of the photoemission yield and the square of the calculated optical field strength are plotted here. The comparison shows good agreement for the lateral distribution and symmetry of the emission pattern. There is, however, some discrepancy as to the strong emission at the far end of the groove, where the experimentally observed photoemission rate is larger than expected from the field strength
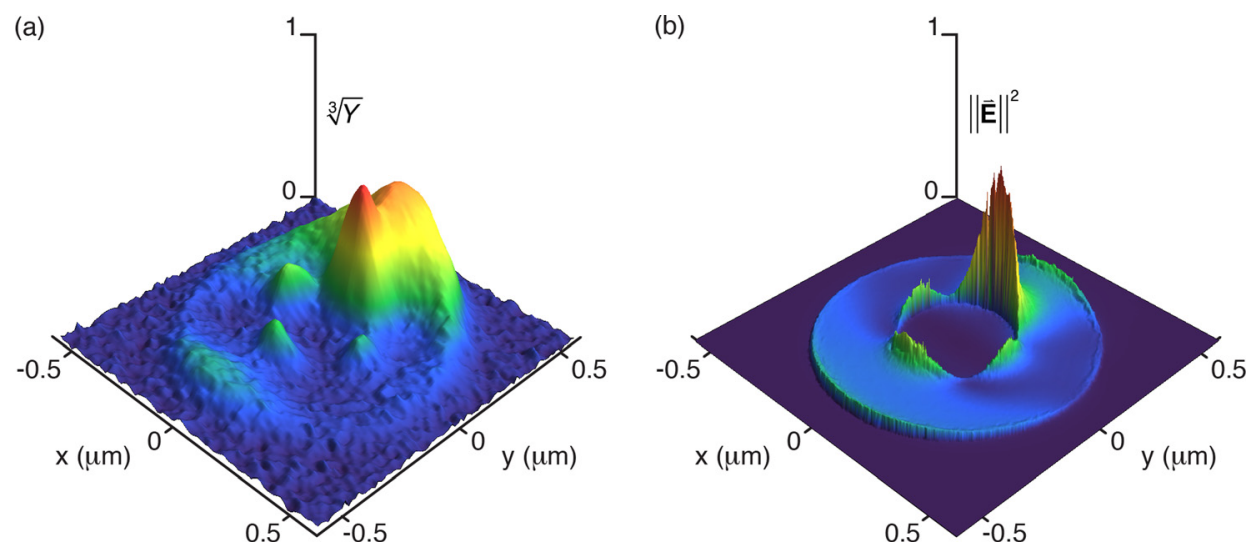

FIG. 2. a) Experimental photoemission micrograph showing the photoemission yield, $\mathrm{Y}^{1 / 3}$. The direction of incidence is in the positive $\mathrm{y}$ direction $60^{\circ}$ from the bottom plane normal as indicated in Fig. 1(c). The polarization is TM. Photoelectron emission is only discernible from within the groove region. Near the inner perimeter a quadrupolar intensity distribution is seen with the main emissions along the plane of incidence. At the outer circumference, a somewhat broader and weaker quadrupolar distribution is seen. The highest emission rates are found at the gap section located at the far end from the laser source. At this location, the electric field vector is nearly perpendicular to the disc surface and the plasmonic excitation is therefore maximized. (b) Calculated optical intensity, $|\mathrm{E}|^{2}$, on a plane inside the metal layer near the ITO interface The intensity distribution shows the same general features and the same symmetry as the photoemission yield. Quantitative differences are attributed to the fact that the photoemission surface is non-planar, while the optical intensity is calculated at a plane. 
TM
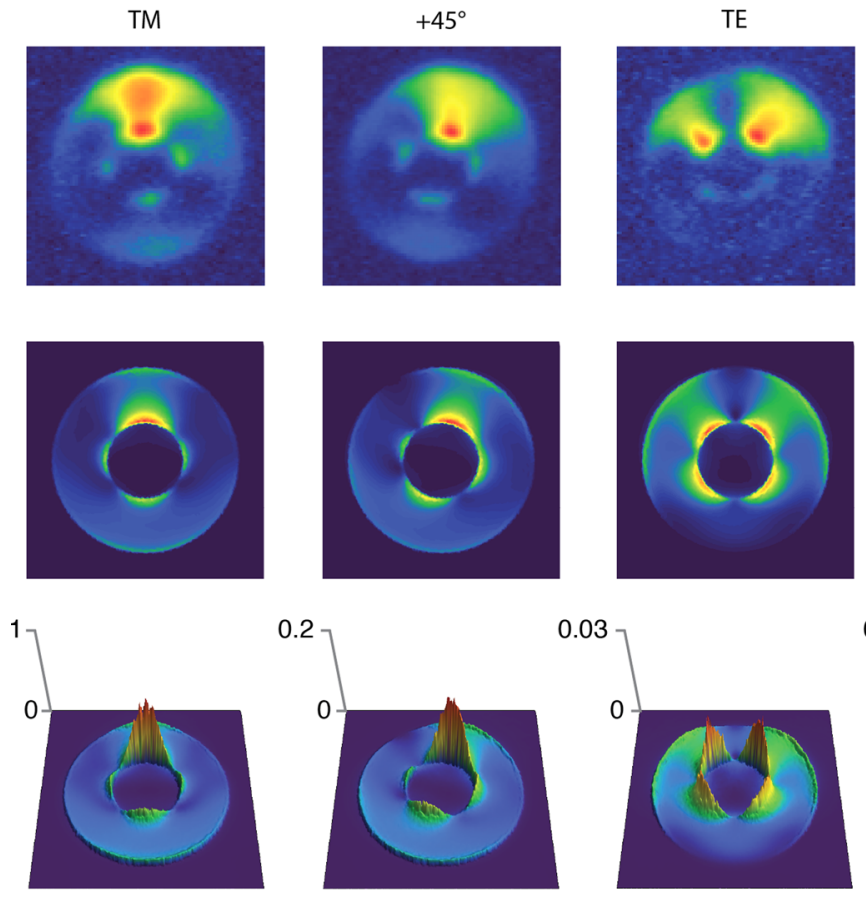
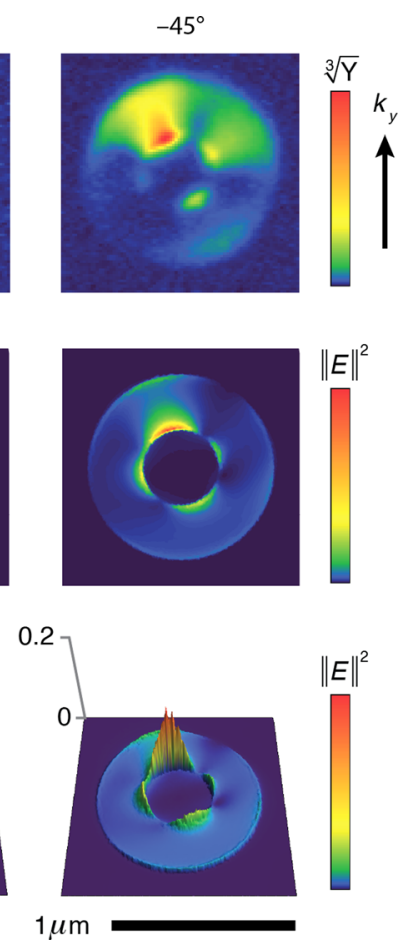

FIG. 3. Upper row: Experimental photoemission micrographs for the polarization dependence of the spatial electron emission distribution for various polarization directions; Center row: Calculated optical intensity patterns $|\mathrm{E}|^{2}$; Bottom row: calculated optical intensity profiles $|\mathrm{E}|^{2}$. calculation. This increased photoemission is likely due to the vertical incidence of the electric field vector of the excitation light on the center disc surface that fosters the plasmonic absorption, and the near vertical incidence of the excitation light on to the curved groove surface. Both effects enhance the emission near in this sector of the ring. The intriguing feature of the arrangement is its the spatial confinement of the electron emission areas. The regions of peaked optical enhancement and photoemission are located at the inner perimeter of the ITO groove and have typical radii of $50-150 \mathrm{~nm}$, substantially less than the wavelength.

We next discuss the possibility of shifting the electron emission areas controllably along the circular groove by changing the polarization of the excitation light. If we assume first that the excitation light falls perpendicular onto the sample surface, then any change in polarization direction would be expected to produce an equal angular rotation of the observed dipole and quadrupole distributions in the device. At a light incidence angle of $60^{\circ}$ from the surface normal, as used here, one expects an angular rotation in the electron emission pattern that follows the polarization change in a similar, but not necessarily identical way. The experimental results and the simulation indeed confirm this expectation as shown in Figure 3. Four different polarization directions are shown. In essence, the rotation angle for the emission pattern is approximately one half of the polarization angle change. The correspondence between experiments and simulations is again very good in this parameter. The device thus allows predictable and precise position control of plasmon-enhanced optical fields and electron emission on a nanometer scale.

Since the polarization angle can be controlled optically, this control possibility is of interest in a wide range of applications, particularly in ultrafast light-controlled optical switches, plasmonic routers, and path selectors. Even on slower time scales, the possibility of spatial control of optical fields and electron emission will be useful in sensors and actuators and in nanoscale electron sources. It is noteworthy that in the device shown here, with sufficiently thin metal layers, the excitation light can, in principle, be applied from both sides of the sample, i.e., through the glass/ITO substrate or from the top side as shown here, with only quantitative differences in the optical and electronic output.

To conclude, we have presented a plasmonic device that allows precise nanoscale motioning of plasmonic optical fields and localized electron emission. It uses polarization control to move the center of plasmonic excitations along a ring-like planar antenna structure. Experimental results and finite-element calculations indicate that a precise and predictable motioning is possible. The device will be useful for plasmonic sensors, actuators, routers, and electron sources.

This research was supported by the US-DOE Basic Science Office under Contract No. DE-FG02-13ER46406.

${ }^{1}$ S. Zeng, D. Baillargeat, H. P. Ho, and K. T. Yong, "Nanomaterials enhanced surface plasmon resonance for biological and chemical sensing applications," Chem. Soc. Rev. 43, 3426 (2014).

${ }^{2}$ S. Linic, P. Christopher, and D. B. Ingram, "Plasmonic-metal nanostructures for efficient conversion of solar to chemical energy," Nat. Mater. 10, 911 (2011).

${ }^{3}$ C. Clavero, "Plasmon-induced hot-electron generation at nanoparticle/ metal-oxide interfaces for photovoltaic and photocatalytic devices," Nat. Photonics 8, 95 (2014).

${ }^{4}$ L. Wu and B. M. Reinhard, "Probing subdiffraction limit separations with plasmon coupling microscopy: Concepts and applications," Chem. Soc. Rev. 43, 3884 (2014).

${ }^{5}$ G. Lozano, D. J. Louwers, S. Rodríguez, S. Murai, O. T. Jansen, M. A Verschuuren, and J. G. Rivas, "Plasmonics for solid-state lighting: enhanced excitation and directional emission of highly efficient light sources," Light: Sci. Appl. 2, e66 (2013).

${ }^{6}$ P. M. Nagel, J. S. Robinson, B. D. Harteneck, T. Pfeifer, M. J. Abel, J. S. Prell, D. M. Neumark, R. A. Kaindl, and S. R. Leone, "Surface plasmon assisted electron acceleration in photoemission from gold nanopillars," Chem. Phys. 414, 106 (2013).

${ }^{7}$ R. Zhang, Y. Zhang, Z. C. Dong, S. Jiang, C. Zhang, L. G. Chen, L. Zhang, Y. Liao, J. Aizpurua, Y. Luo, J. L. Yang, and J. G. Hou, "Chemical 
mapping of a single molecule by plasmon-enhanced Raman scattering," Nature 498, 82 (2013).

${ }^{8}$ R. Könenkamp, R. C. Word, J. Fitzgerald, A. Nadarajah, and S. Saliba, "Controlled spatial switching and routing of surface plasmons in designed single-crystalline gold nanostructures," Appl. Phys. Lett. 101, 141114 (2012).

${ }^{9}$ R. Könenkamp, R. C. Word, G. F. Rempfer, T. Dixon, L. Almaraz, and T. Jones, " $5.4 \mathrm{~nm}$ spatial resolution in biological photoemission electron microscopy," Ultramicroscopy 110, 899 (2010).

${ }^{10}$ R. C. Word, J. P. S. Fitzgerald, and R. Könenkamp, "Electron emission in the near-field of surface plasmons," Surf. Sci. 607, 148 (2013).
${ }^{11}$ L. Novotny and N. F. van Hulst, "Antennas for light," Nat. Photonics 5, 83 (2011).

${ }^{12}$ K. Li, M. I. Stockman, and D. J. Bergman, "Self-similar chain of metal nanospheres as an efficient nanolens," Phys. Rev. Lett. 91, 227402 (2003).

${ }^{13}$ F. P. Schmidt, H. Ditlbacher, U. Hohenester, A. Hohenau, F. Hofer, and J. R. Krenn, "Universal dispersion of surface plasmons in flat nanostructures," Nat. Commun. 5, 3604 (2014).

${ }^{14}$ J. P. S. Fitzgerald, R. C. Word, and R. Könenkamp, "Subwavelength visualization of light in thin film waveguides with photoelectrons," Phys. Rev. B 89, 195129 (2014). 